\title{
A similarity solution for the flow and heat transfer over a moving permeable flat plate in a parallel free stream.
}

\begin{abstract}
This paper presents both a numerical and analytical study in connection with the steady boundary layer flow and heat transfer induced by a moving permeable semi-infinite flat plate in a parallel free stream. Both the velocities of the flat plate and the free stream are proportional to $\mathrm{x} 1 / 3$. The surface temperature is assumed to be constant. The governing partial differential equations are converted into ordinary differential equations by a new similarity transformation. Numerical results for the flow and heat transfer characteristics are obtained for various values of the moving parameter, transpiration parameter and the Prandtl number. Approximate analytical solutions are also obtained when the suction or injection parameter is very large. It is found that dual solutions exist for the case when the fluid and the plate move in the opposite directions.
\end{abstract}

Keyword: Heat transfer; Approximate analytical solutions; Dual solutions; Flat plate; Flow. 